\title{
Research on Innovative Design of Bathroom Furniture Based on Olfactory Experience
}

\author{
Kaiqin Feng ${ }^{1,2}$, Ming Chen ${ }^{1,2, a,{ }^{*}}$ and Jianhua $\operatorname{Lyu}^{1,2, \mathrm{~b}}$ \\ ${ }^{1}$ Forestry College, Sichuan Agricultural University, Chengdu 611130, China; \\ ${ }^{2}$ Key Laboratory of Wood Industry and Furniture Engineering of Sichuan Provincial Colleges and \\ Universities, Sichuan Agricultural University, Chengdu 611130, China. \\ achenming@sicau.edu.cn, bljh@sicau.edu.cn
}

Keywords: Olfactory experience, Bathroom furniture, fragrance, Innovative design.

\begin{abstract}
This paper, starting from the background of the olfactory experience, analyzes the necessity of the innovative design of the olfactory experience in the bathroom furniture, expounds the concept of the olfactory sensation and olfactory experience, and summarizes the relationship between the olfactory experience and the human's psychological and behavior. This paper also discusses the idea and method of innovative design of sanitary ware based on olfactory experience, and by making an empirical demonstration, it designs two bathroom furniture with fragrance occurrence function, which provides a new idea for the design of olfactory experience in furniture products.
\end{abstract}

\section{Introduction}

Bathroom furniture is an integral part of the bathroom space and an indispensable part of people's lives, mainly for people to wash and dress. In a broad sense, the functions of bathroom furniture include material function and spirit function. Its spirit function comes from the Human sensory nature to the bathroom furniture. Given that the person's feeling is generally considered to have 5 kinds that is so-called "five Senses", referring to the vision, the tactile sense, the smell, the taste and the hearing, the visual impact on the mental function of bathroom furniture is the most direct[1]. But the nature of the feeling should not only be limited to the visual level, other human sensory characteristics also, to varying degrees, affect the realization of the spiritual function of bathroom furniture, and then affect people's emotional and even emotional experience[2].With the development of social economy, people's consumption level, consumption consciousness and aesthetic concept are constantly changing. It is particularly urgent to combine the sensory design based on olfactory experience with the design of bathroom furniture.

\section{Smell and Olfactory experience}

About the relationship between the senses and the image, Kenya Hara once said:" Man is not just a sensory organ combination, but also a sensitive memory regeneration device, and can reproduce a variety of images according to memory in the mind [3]." The essence of smell is the sensation caused by the chemical molecules stimulating the olfactory receptor, which is one of the oldest senses in the process of evolution [4]. People rely on the smell to achieve the perception of smell, and different odors to exert a differential effect on the human body.

The olfactory experience is to add odor to the design of the furniture based on the human's olfactory physiological characteristics, making people feel useful in the use of the product. The effectiveness of olfactory experience is closely related to the type, concentration and persistence of the odor. In general, the research and use of the olfactory experience is inadequate in furniture design which pays more attention to the rational use of color, form, texture and other elements, emphasizing on the visual experience and tactile experience of furniture products. From the perspective of multiple sensory design concepts and the functional spiritual needs of people for furniture, in addition to the visual experience and tactile experience, olfactory experience is more potential and more feasible in 
the design of furniture products than auditory experience and taste experience. The addition of olfactory experience can not only enriches the functional spirit of furniture products, but also enhances the added value of furniture products, which meets consumers' increasingly individualized consumption [5].

\section{The Relationship between Olfactory Experience and Human Behavior \& Psychology}

The olfactory experience is not insignificant. And the smell has a direct connection with people's psychology and behavior. Odor is the most important factor that affects people's psychological and behavioral choices. The smell can affect our mood and motivation, and then affect our behavior, which is more likely to cause physical responses than visual. The study shows that "the nerves in the human brain that deal with the sense of smell are closely linked to the central nerve system in charge of emotional control, and there is no doubt that the smell will certainly affect people's emotions and feelings [6]."

In general, the odor can be divided into three kinds: positive, negative and neutral. Positive smells can make people feel comfortable and reminiscent of good things. The act is to remember or strengthen the sense of smell, that is, to approach the source of smell or to remember the cause of the smell [7]. Some positive smells also help with people's health. For example, a variety of air fresheners sold in the supermarket is to make people's environment smell clean, refreshing and pleasant. And the fragrant smell can help people to have an optimistic mood and to cultivate positive emotions. Negative smells are unpleasant smells. Negative smells tend to make people irritable, anxious, upset, and nervous. So when unpleasant odors appear, people will escape or try their best to remove odors. Odors can be taken as a warning. For instance, once the kitchen gas leaks, people will immediately realize the dangerous mood, and move to the ventilated, safe zone [8].

Human senses are interrelated and they influence each other and determine people's behavior. The smell can affect our emotions and motives, and thus affect our behavior. Although the sense of smell and emotion are two different concepts, the two are closely linked. Compared with other sensory, the smell has emotional characteristics, and is similar to the emotional classification. Odors will also bring positive, negative, neutral and other emotional labels. Olfactory stimulation can induce different types and degrees of emotional state. Conversely, changes of mood can also affect the sense of smell.

\section{Innovative Design of Bathroom Furniture based on Olfactory Experience}

Based on olfactory experience, innovative design of bathroom furniture, according to the relationship between smell and human emotion, psychology and behavior, is regarding bathroom furniture as the carrier to add odors in the bathroom furniture, so that users can have new experience. With the development of science and technology, more and more chemical materials are used in the manufacturing industry, and odors given off by some of them make consumers feel worried and fear. As a new subject, the olfactory experience design pays attention to the smell of bathroom furniture and the influence on the space. It reflects the humanistic concern of "designing for the people [7]".

\subsection{Increasing the Good Olfactory Experience}

Increasing good olfactory experience means adding new scent elements to the original product or environment. In the bathroom space, the existence of bathroom furniture can meet people's needs of storage, dressing and so on. However, it can not only meet the basic needs of consumers, but also improve the satisfaction during the usage by increasing the olfactory experience function in the bathroom furniture. Moreover, the limitation and closeness of the bathroom space is conducive to the efficiency of odor emission and the effectiveness of olfactory experience.

The reasonable choice of furniture material is the precondition of producing a good smell experience. Especially the wood material itself can release the substance with aromatic odor. For example, Lobular Rosewood has a substance known as "wood oxygen", which can emit a faint fragrance, making people calm and nerve-soothing [9]. The use of plate furniture in the bathroom 
furniture is larger. But it is difficult to distribute the volatile and harmful substances such as formaldehyde in panel furniture because of its small space and poor air circulation, thus affecting people's health seriously. Therefore, it can reduce or isolate harmful volatile and bring good smell experience via the selection of qualified materials, the implementation of strict technology.

With the development of science and technology, most of the odor sources removing the odorous substances of furniture itself are artificially manufactured. Spices are made in a variety of ways, both in a solid state and in a liquid state. For example, in order to improve the durability of the fragrance of the textile, the essence is made into microcapsules to realize the slow release of fragrance, and then the essence microcapsules are fixed to the fabric through proper adhesive or other finishing agents [10]. In the design of bathroom furniture, it can get better olfactory experience by adding odor source and odor-volatile device and combining artificial fragrance with furniture. Among them, the odor source device can utilize the odor volatile characteristics. To the bathroom furniture design, it adds the material-source design with volatile odor. It meets the needs of different people and their preference to obtain different olfactory experience. Odor-volatile devices are able to use wind, heat, and sound waves and other technologies to make odors spread and evaporate better. The combination of the two devices can make the odorant in the odor source device evaporate faster. At the same time, the source of odor can be changed according to different purposes and personal preferences in the design process. This approach is also consistent with the trend of personalized design.

\subsection{Eliminating bad olfactory experience}

Eliminating the bad olfactory experience that is, removing discordant and unpleasant odor elements in furniture or the environment. Bathroom space is a place where people use a higher frequency of life. It's easy for bacteria to breed because of the humid environment and the non-circulating air. At the same time, a larger odor will be produced during using, bringing bad olfactory experience.

Odor elimination device can be added to eliminate the bad smell in the bathroom furniture design, so that people's sense of smell could return to normal. Milliken has developed a textile technology-visa endurance, which absorbs the smell of the human body and suppresses the smell effectively. The technology mainly uses silver ion antimicrobial principle to achieve sterilization and ventilation, resulting in control and absorption of odor characteristics. Activated carbon is also the most commonly used odor-killing substance in daily life. And it has strong adsorption capacity owing to the large surface area which can make full contact with gas and rich micropores of small carbon particles. Only when adsorption materials are use to eliminate or absorbing bad odor can it meets the requirements better.

\section{Design Demonstration}

The design validation is conducted by the analysis of the relationship between olfactory experience and human psychology behavior. And innovative design ideas and approaches are based on research. For bathroom furniture, its space is usually bathroom space which is the first space after we get up in the morning. A bathroom space with good olfactory experience is helpful for the mood of the day. We all know that bathroom space often has some peculiar smell, even some odor molecules will remain on the body. In order to solve the above problems, the author designs two bathroom furniture, which can give users a good olfactory experience.

The first bathroom furniture is a bathroom mirror with scented functions. The appearance and visible part of this bathroom mirror is shown in Figure 1. 

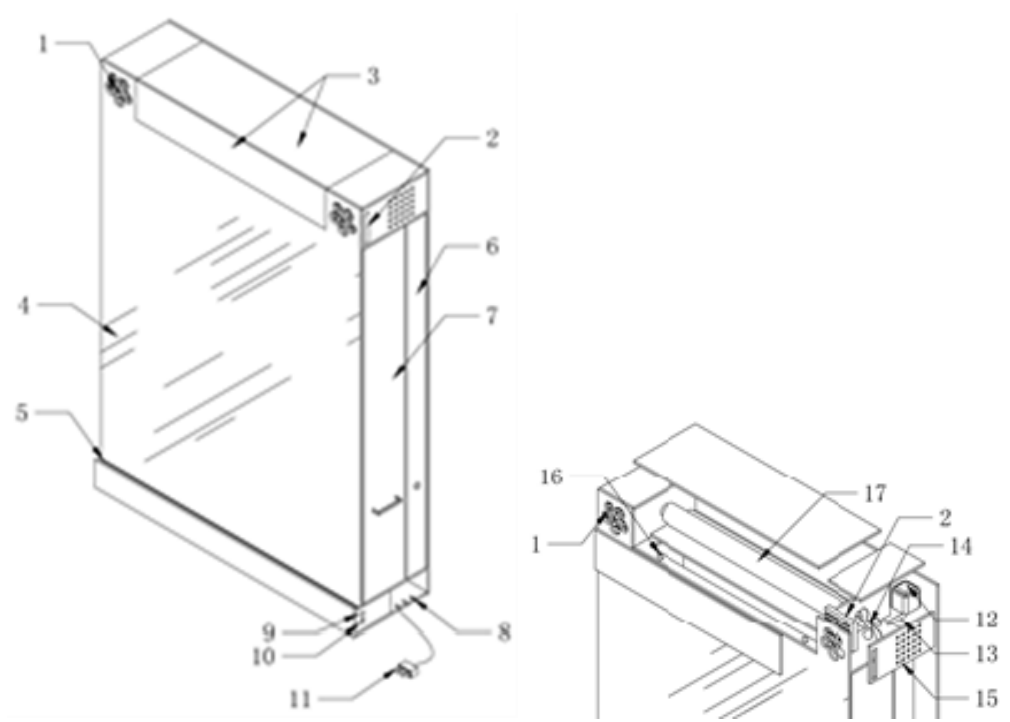

1 is aroma outlet 2 is spice furnishing box 4 is mirror

3 is transparent plastic board 6 is storage cabinet (small)

7 is storage cabinets (large ) 9 is button 11 is two-hole plug

12 is the motor of fragrance machine 13 is the shaft of fragrance machine, which is connect to 12 the motor of fragrance machine and 14 the fan blade of fragrance machine 14 is the fan blade of

fragrance machine 15 is the air inlet of fragrance machine

Fig. 1 bathroom mirror with

Fig. 2 the interior of fragrance machine

fragrance generating function[11]. and the function parts of lamp[11]

The interior of fragrance machine and the function parts of lamp is shown in Figure 2. In the case of power plug, fragrance machine will start, then fragrance mechanical and electrical motivation will work. Through fragrance machine shaft driving fan leaf rotation, fragrance machine fan will blow out the aroma of the tuyere through the Spice furnishing box, so that the bathroom space will be full of fragrance. When the person is close to the infrared sensor, the lamp tube will send out lights and then automatically starts and illuminates the outside through the transparent plastic board. It's easy to replace spices according to the user's preferences and the use of time. Odor source device, that is spice, has been added in the bathroom mirror in this design. And it's volatilized by wind, producing a positive smell. People's unpleasant psychological impression of bathroom space will be solved, making them happy and getting good olfactory experience.
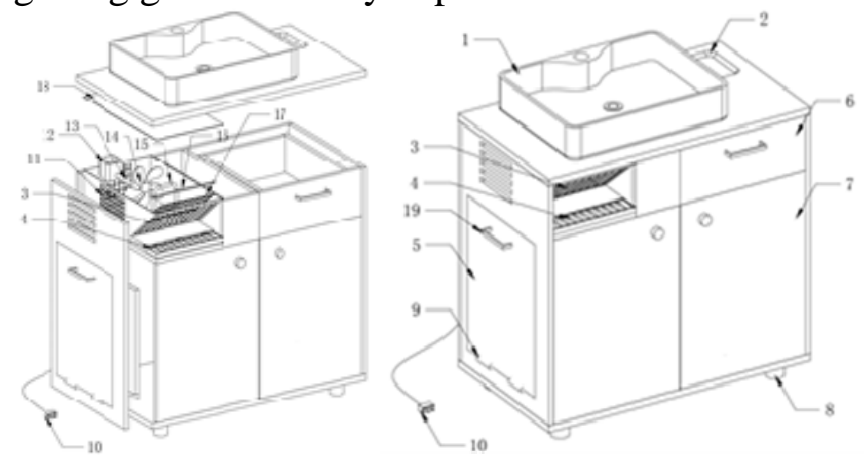

fragrance occurrence function [12] components of Hand-dryer [12]

1 is wash celestial basin 3 is the air outlet of the Hand-dryer 4 is spice furnishing box

10 is two-hole plug 11 is air inlet 12 is motor 14 is the fan blade

13 is the shaft, which is connect to 12 the motor and 14 the fan blade

15 is resistance wire 16 is infrared sensor 17 is wire

Fig. 3 bathroom cabinet with

Fig. 4 the internal functional

The second bathroom furniture is a bathroom cabinet with fragrance occurrence function. Figure 3 is the shape and appearance of this bathroom mirror. It can not only scent, but also dry hands. Figure 4 is the internal functional components of Hand-dryer. Hot air device is designed in the bathroom cabinet, and the box for spices is designed in the outlet. When people put their hands after washing on 
the air outlet, it will induce switch, generating hot air. When the infrared sensor senses hands, the motor will work. Through the rotating shaft to drive the fan leaf rotation, fan leaves the wind through the resistance wire heating. And through the wire mesh storage resistance wire heat, the wind will be evenly heated, and then come from the outlet. Hot air can make the spices volatile and diffuse easily. It can not only leave fragrance in hand, but also make people have a good olfactory experience of bathroom space. Odor source device, that is spice, has been added in the bathroom cabinet in this design. The wind and heat can volatile fragrance effectively through the odor volatile device. The heat can also reduce the humidity of the bathroom space, bringing positive odor and refreshing feelings.

\section{Summary}

With the progress of society, people's psychological demand for products is getting higher and higher. Only by expanding design ideas can we meet the realistic and potential demands of consumers. This is the necessary way for innovative design of furniture products. Innovative design of bathroom furniture based on olfactory experience creates a good olfactory experience for people, affecting people to have a good psychological feeling. It also affects people's activities, for example, making people work more energetically or letting the tired body relaxed.

At present, the development and research of olfactory furniture in China fails to form a relatively mature, stable research and industrial system. And it is basically in the theoretical stage or the state of small batch production. Due to the lack of experience, technology and equipment, it is a certain challenge for furniture designers in the design of olfactory furniture. But with the development of science and the desire for olfactory furniture, designers will be able to overcome many difficulties and will design practical, safe, beautiful and easy-to-use olfactory furniture.

\section{Acknowledgments}

This project is supported by Key Laboratory of Wood Industry and Furniture Engineering of Sichuan Provincial Colleges and Universities.

\section{References}

[1]. Jinlin Zou, Yiqing Peng. Application of Auditory in Product Design.Packaging Engineering. Vol. 35 (2014) No. 8, p. 105-108.

[2]. Jiuping Cheng, Shiguo LI, Jian Zhang. Application Research of Emotional Experience Design Based on Motive of Stimulus Seeking. Packaging Engineering. Vol. 34 (2013) No.14, p. 68-71.

[3]. Kenya Hara. Design of Design. Shandong People’s Publishing House, 2007, p. 75.

[4]. Wen Zhou, Feng Guo. Olfactory Perception and Its Interplays with the Emotional Svstem. Advances in Psychological Science. Vol. 20 (2012) No. 1, p. 2-9.

[5]. Ming Chen, Shangguan Li, Chunxiao Li, Kaiqin Feng, Jianhuan Lyu. Furniture Product Innovative Design and Empirical Based on Smell Experience. Packaging Engineering. Vol. 37 (2016) No. 4, p. 92-95.

[6]. Youhao Zhang. Olfactory marketing and inducing desire. Technology Daily. 2000-08-13(4).

[7]. Rina Sa: Design of Olfactory Experience in Household Goods (Master Thesis, Central Academy of Fine Arts, China 2010). p. 5.

[8]. Yanchun Xu: Application of olfactory perception in the bonks design experience (Master Thesis, Shandong University of Art and Design, China 2013). p. 4-5.

[9]. Xi Wu, Jianhua Lyu, Ming Chen. Traditional Chinese Medicine Regimen Efficacy of Hongmu Furniture from the Hongmu Culture. Furniture. Vol. 35 (2014) No. 4, p. 99-101.

[10]. Hongdan Liu, Chengjie Shang. Health Care Efficacy of Aromatherapy and Its Application in Textiles. Knitting Industries. No. 9 (2012) p. 42-44. 
[11]. Sichuan Agricultural university. Multifunctional bathroom cabinet. Patent. China: ZL201520071810.6, 2015-06-10.

[12]. Sichuan Agricultural University. Multifunctional bathroom cabinet. Patent. China: ZL201420792391.0, 2015-04-08. 